\title{
miRNA expression profile in human osteosarcoma: Role of miR-1 and miR-133b in proliferation and cell cycle control
}

\author{
CHIARA NOVELLO ${ }^{1}$, LAURA PAZZAGLIA ${ }^{1}$, CHIARA CINGOLANI $^{1}$, AMALIA CONTI $^{1}$, IRENE QUATTRINI $^{1}$, \\ MARIA CRISTINA MANARA ${ }^{1}$, MAURO TOGNON ${ }^{2}$, PIERO PICCI $^{1}$ and MARIA SERENA BENASSI ${ }^{1}$ \\ ${ }^{1}$ Laboratory of Experimental Oncology, Rizzoli Orthopaedic Institute, I-40136 Bologna; \\ ${ }^{2}$ Department of Morphology and Embryology, Section of Cell Biology and Molecular Genetics, \\ School of Medicine and Center of Biotechnology, University of Ferrara, I-44100 Ferrara, Italy
}

Received June 29, 2012; Accepted September 24, 2012

DOI: 10.3892/ijo.2012.1717

\begin{abstract}
RNA profile deregulation affecting downstream signaling pathways activates endpoints that represent potential biomarkers for prognosis and treatment of tumor patients. In the past 20 years conventional therapy for osteosarcoma (OS) reached a survival plateau, highlighting the need for new therapeutic approaches. In this study, microarray unsupervised and supervised analysis identified, respectively, 100 and 40 differentially expressed miRNAs in OS samples with different grades of malignancy compared to normal bone. When analyzing low-grade and high-grade OS by unsupervised analysis, 12 miRNAs were found to be differentially expressed. Real-time PCR performed on a larger series of OS confirmed a significant lower expression of miR-1, miR-133b and miR-378* in tumors with respect to control, also showing lower mRNA levels in 31 high-grade OS than in 25 low-grade and in metastatic versus non-metastatic patients. We demonstrated that miR-1 and miR133b were downregulated in OS cell lines compared to normal osteoblasts. Secondly, by transfection with miRNA precursor molecules, we demonstrated that the ectopic expression of miR-1 and miR-133b in U2-OS cell lines significantly reduced cell proliferation and MET protein expression and negatively regulated cell invasiveness and motility in a short-term assay. Cell cycle distribution revealed block in $\mathrm{G}_{1}$ and delay of cell cycle progression associated with increased apoptosis in miR-1- and miR-133b-transfected cells, respectively. Our data assessed specific miRNA profiling deregulation in OS clinical samples and suggest that the expression of miR-1 and miR-133b may control cell proliferation and cell cycle through MET protein expression modulation.
\end{abstract}

Correspondence to: Dr Maria Serena Benassi, Laboratory of Experimental Oncology, Rizzoli Orthopaedic Institute, Via di Barbiano 1/10, I-40136 Bologna, Italy

E-mail: mariaserena.benassi@ior.it

Key words: microRNA microarray, osteosarcoma, cell proliferation, apoptosis, Met expression

\section{Introduction}

Osteosarcoma (OS) is a rare malignant bone neoplasm for which biologic and pathologic information are still largely incomplete. OS has multiple genetic risk factors (1) and is characterized by complex chromosomal abnormalities and genetic cell heterogeneity that in over $70 \%$ provide a complex karyotype and drug resistance (2). MicroRNAs (miRNAs) are endogenous non-coding RNAs of 19-24 nucleotides that interacting with the 3' untranslated region (UTR) of mRNA target induce mRNA cleavage when pairing is complete and protein synthesis repression when pairing is incomplete. The relationship between miRNAs and their targets shows combinatorial complication, in terms of both target multiplicity and signal integration (3). MiRNAs are differentially expressed in relation to the developmental state, cell type and tissue (4-6). In literature, there are approximately 1,000 miRNA molecules per cell, with some cells exceeding 50,000 molecules (7). miRNA expression follows a dynamic range and this underscores the regulatory functional importance of miRNAs (3). Recent studies indicate that miRNAs called 'oncomirs' can function either as tumor suppressors or as oncogenes (8).

Interconnection between miRNA and cancer is even more evident when analyzing the genomic location of known miRNA genes, more than $50 \%$ are in cancer-associated genomic regions or in fragile sites (8-10). To date, more than 1,100 miRNAs have been identified in humans and the number of microRNA implicated in human cancer is increasing. Recently distinct miRNA expression signatures have been proposed as diagnostic and prognostic markers for various types of human cancer $(5,8)$.

Our recent results demonstrated miR-93 overexpression involved in cell growth and invasion through downstream regulation of E2F1 transcription factor (11). Other studies recognized miR-34 as a direct target of p53 (12). A study on 27 OS samples identified miRNAs discriminating good and poor responder patients treated with ifosfamide. These results showed overexpression of miR-92a, miR-99b, miR-193a-5p, miR-422a and underexpression of miR-132 in the good responders group (2). In this study, miRNA expression profile performed on human OS specimens and cell lines 
aimed to recognize new molecules involved in OS progression through information on regulation of genes controlling cell proliferation, invasion and migration.

\section{Materials and methods}

Tumor specimens. Primary tumor samples from 56 patients with osteosarcoma were referred to the Rizzoli Orthopaedic Institute and diagnosed by expert pathologists. A total of 31 were high-grade tumors and 25 were low-grade. Thirty-one patients were males and 25 females with a mean age of 23 years. At a minimum follow-up of 5 years, 21 patients had lung metastases and 15 died of disease (Table I). For each specimen frozen material was available and in all specimens the percentage of tumor cells estimated after hematoxylineosin staining was equal or more than $90 \%$. Thirteen normal bone from non-cancer patients were used as control. The research protocol was approved by ethic committees of the Rizzoli Institute where the tumor samples were collected and all patients provided appropriates informed consent.

Cell lines and culture conditions. Human OS cell lines U2-OS, MG-63 and SAOS, and normal osteoblast cells were obtained from the American Type Culture Collection (ATCC, Rockville, MD, USA) and cultured in Dulbecco's modified Eagle's medium (DMEM) supplemented with $10 \%$ fetal bovine serum (FBS), L-glutamine $(2 \mathrm{mM}), 100 \mathrm{U} / \mathrm{ml}$ penicillin and $100 \mu \mathrm{g} / \mathrm{ml}$ streptomycin (Invitrogen, Carlsbad, CA, USA) at $37^{\circ} \mathrm{C}$ in a $5 \% \mathrm{CO}_{2}$ humidified incubator.

RNA extraction. Total-RNA was extracted from cell lines and frozen tissues $(150 \mathrm{mg})$ using TRIzol Reagent (Invitrogen) according to the manufacturer's protocol and stored at $-80^{\circ} \mathrm{C}$ in RNAsecure reagent (Ambion Inc., Austin, TX, USA). Concentration of total-RNA was measured with spectrophotometer and the 260/280 ratio of RNA was 1.8 . Purity and quality were identified by a denatured gel electrophoresis.

MicroRNA array profiling. Total-RNA of 6 low-grade, 6 high-grade OS and 6 healthy tissues was profiled by Exiqon (www.exiqon.com) using miRCURY LNA array vs 11.0 hsa, mmu and rno (13). Briefly, after RNA quality control performed by Bioanalyser 2100 and RNA measurement on the Nanodrop instrument, using the miRCURY ${ }^{\mathrm{TM}}$ Hy3/Hy5 Power Labeling kit (Exiqon, Vedbaek, Denmark), experimental samples and a reference sample were labeled in separate reactions with $\mathrm{Hy} 3$ and Hy5, respectively. Labeling efficiency was evaluated by analyzing the signals from control spike-in capture probes. Labeled experimental and reference samples were combined, denatured, and hybridized to microarrays at $56^{\circ} \mathrm{C}$ for $16 \mathrm{~h}$. Low-stringency and high-stringency washes were carried out and the microarrays dried. Images were acquired using the Axon ${ }^{\circledast}$ GenePix 4000B scanner and GenePix software. The data was pre-processed and normalized using the global locally weighted scatterplot smoothing procedure (14).

miRNA expression analysis by real-time PCR. Analyses were performed in 56 OS tissues ( 31 high- and 25 low-grade), 13 normal samples, and in OS cell lines and osteoblasts. Reverse transcription and real-time PCR (RT-PCR) were carried out following TaqMan MicroRNA Assay Protocol (Applied Biosystems, Foster City, CA, USA) and the expression of miR-1 (miRNA assay no. 002222), miR-133b (miRNA assay no. 002247) and miR-378* (miRNA assay no. 000567) were quantified using $2^{-\Delta \Delta \mathrm{Ct}}$ comparative method (Applied Biosystems, User Bulletin no.2 P/N 4303859) and normalized using RNU44 as endogenous reference (miRNA assay no. 001094). Mesenchymal stem cells (MSC) (ATCC) were used as calibrator for cell lines, while normal osteoblasts resulted the most adequate calibrator for our human OS series. Each miRNA was considered more expressed when the value calculated with the method $2^{-\Delta \Delta C t}$ was higher than the value $1.5 \pm \mathrm{SD}$ or less expressed when the value was below $0.5 \pm \mathrm{SD}$.

Statistical analysis. Unsupervised and supervised hierarchical cluster analysis was performed on $\log 2$ (Hy3/Hy5) ratios in normal and tumor tissues. P-value for unsupervised analysis was set at $\mathrm{p}<0.05$, for supervised analysis was set at $\mathrm{p}<3.95 \mathrm{E}-5$. miRNA expression data are shown as median $(\mathrm{m})$ for their strong non-Gaussian distribution. Non-parametric Mann-Whitney $\mathrm{U}$ test was performed to compare miRNA expression profile in unpaired samples; $p$-values were set at $<0.05$.

Transfection of miR-1 and miR-133b precursor molecule. OS cells were seeded at a density of $1.5 \times 10^{5}$ per well in 6 -well plates in $2 \mathrm{ml}$ of complete medium containing $10 \%$ FCS for $24 \mathrm{~h}$. Transfections were performed using reagent (Lipofectamine 2000, Invitrogen). For each well miR-1 precursor (cod. PM10617, Ambion Inc.), miR-133b precursor (cod. PM10029, Ambion Inc.) or negative control precursor miRNA (scramble) (cod. AM17110, Ambion Inc.) was transfected into U2-OS cells. Pre-miR miRNA precursor molecules are small, chemically modified, double stranded RNA molecules designed to mimic endogenous mature miRNAs once properly transfected and expressed by recipient cells.

The mixture Lipofectamine-miRNA (miR-1, $50 \mathrm{nM}$; miR-133b, $100 \mathrm{nM}$ ) was then administered to cells at $37^{\circ} \mathrm{C}$ in presence of serum-free medium up to $72 \mathrm{~h}$. Optimal transfection conditions were obtained by using Lipofectamine $2000(1 \mathrm{mg} / \mathrm{ml}$, Invitrogen) at a weight ratio miRNA-transfectant of 1:1. Transfection efficiency was monitored by flow cytometry (FACSCalibur, BD Biosciences, San Jose, CA, USA) using $\mathrm{Cy}^{\mathrm{TM}} 3$ and $\mathrm{FAM}^{\mathrm{TM}}$ dye-labeled Pre-miR negative controls (cod. AM17120, Ambion Inc.) and by RT-PCR using $\Delta \mathrm{Ct}$ values. All in vitro assays were performed at 24 , 48 and $72 \mathrm{~h}$ of transfection using scramble and non-treated cell lines (U2-OS wt) as negative controls. All experiments were repeated at least three times.

Cell growth assay. The number of adherent, viable cells was assessed microscopically using an improved Neubauer hemocytometer and viability was assessed as the percentage of cells that excluded $0.2 \%$ trypan blue. After 24, 48 and $72 \mathrm{~h}$ from transfection, the cells were washed once with 1X Dulbecco's phosphate buffered saline (PBS), harvested by trypsinization and cell number was determined using trypan blue. 
Migration assay. Cell migration assay was carried out using Transwell Permeable Support (Corning Incorporated, Corning, NY, USA). After transfection U2OS cells were carefully transferred on the top chamber of each transwell apparatus at a density of $1 \times 10^{6}$ per $\mathrm{ml}$ (100 $\mu \mathrm{l}$ per chamber). Cells were allowed to migrate for 24,48 and $72 \mathrm{~h}$ at $37^{\circ} \mathrm{C}$. Cells that had penetrated to the bottom side of the membrane were then fixed in methanol, stained using hematoxylin and counted at microscope.

Invasion assay. Cell invasion was analyzed by using Cultrex 24-well BME Cell Invasion Assay (Trevigen Inc., Gaithersburg, MD, USA) according to standard procedures. Briefly, after transfection, $10^{3}$ OS cells were seeded in $100 \mu 1$ serum-free media into the upper wells previously coated with Matrigel basement extract, and $500 \mu \mathrm{l}$ of media were added in the bottom wells. After 24, 48 and $72 \mathrm{~h}$ of $\mathrm{CO}_{2}$ incubation at $37^{\circ} \mathrm{C}$, the non-invasive cells on the upper surface were removed and the cells migrated to the lower surface were fixed in $500 \mu \mathrm{l}$ of Cell Dissociation Solution/Calcein-AM, incubated al $37^{\circ} \mathrm{C}$ in $\mathrm{CO}_{2}$ incubator for $1 \mathrm{~h}$ and quantified by fluorimetric analysis (485 excitation, $520 \mathrm{~nm}$ emission).

Apoptosis. Apoptotic and necrotic cell death were analyzed by double staining with fluorescein isothiocyanate (FITC)-conjugated Annexin V and PI, in which Annexin V bound to the apoptotic cells with exposed phosphatidylserine, while PI labeled necrotic cells with membrane damage using an Annexin V-FITC apoptosis detection kit (MEBCYTO Apoptosis kit, MBL International, Woburn, MA, USA). The green (FL1) and red (FL2) fluorescence of Annexin/PI-stained live cells and PI-stained fixed cells was analyzed with a FACSCalibur flow cytometer (BD Biosciences) using a peak fluorescence gate to exclude cell aggregates during cell cycle analysis. The numbers of viable (Annexin $\left./ \mathrm{PI}^{-}\right)$, apoptotic $\left(\right.$Annexin $\left.{ }^{+} / \mathrm{PI}^{-}\right)$and necrotic $\left(\right.$ Annexin $\left.{ }^{+} / \mathrm{PI}^{+}\right)$cells were determined with CellQuest Software (BD Biosciences). According to the protocol, after 24, 48 and $72 \mathrm{~h}$ from transfection, adherent cells were briefly trypsinized, detached, and combined with floating cells from the original growth medium, centrifuged and washed twice with phosphate-buffered saline (PBS). Cells were resuspended in $500 \mu \mathrm{l}$ of staining solution containing FITC-conjugated Annexin V antibody and propidium iodide (PI). After incubation on ice for $30 \mathrm{~min}$, cells were analyzed by flow cytometry. Basal apoptosis and necrosis were identically determined on untreated cells.

Cell cycle analysis. Flow cytometric analyses were performed to define cell cycle distribution for transfected and not transfected cells. After 24, 48 and 72 h from transfection, cells were harvested by trypsinization and fixed with $70 \%$ ethanol. Cells were stained for total DNA content with a solution containing $20 \mu \mathrm{g} / \mathrm{ml}$ propidium iodide. Cell cycle distribution was then analyzed with a FACScalibur flow cytometer (BD Biosciences).

Met gene expression by real-time PCR. Reverse transcription of mRNA from U2-OS wt, U2-OS transfected with miR-1, miR-133b and scramble was carried out in $100 \mu \mathrm{l}$ final volume from 400 ng total-RNA using High Capacity cDNA Archive kit (Applied Biosystems) according to manufacturer's instructions. Quantitative RT-PCR was performed using ABI 7900 sequence detection system (Applied Biosystems) according to manufacturer's protocol. Met gene was quantified by $\Delta \mathrm{Ct}$ method using TaqMan Expression Assays (Hs01565584_m1) (Applied Biosystems) and normalized to a housekeeping ACTB (Hs99999903_m1 gene; TaqMan Expression Assays, Applied Biosystems).

Western blot analysis. According to standard procedures, $50 \mu \mathrm{g}$ of protein extracts from cell lysate of U2-OS wt, U2-OS transfected with miR-1, U2-OS transfected with miR-133b and scramble were prepared and analyzed by 6\% SDS-PAGE. Western blot analysis (WB) was performed by using anti-MET(C-28) (Santa Cruz Biotechnology, Santa Cruz, CA, USA) diluted 1:200. The signal was visualized by Immobilon Western Chemiluminiscent HRP substrate (Millipore, Billerica, MA, USA) and quantified by densitometric analysis using GS-800 imaging densitometer and Quantity One software (Bio-Rad, Hercules, CA, USA). A rabbit anti-actin antibody (Sigma Chemical Co., St. Louis, MO, USA) was used as control.

\section{Results}

Microarrays. In the first step, we analyzed total-RNA from 6 high-grade OS (patients 1-6; Table IA), 6 low-grade OS (patients 1-6; Table IB) and 6 normal bone samples from non cancer patients used as control. The unsupervised hierarchical clustering, performed on $\log 2$ (Hy3/Hy5) ratios, revealed 100 miRNAs with the highest standard deviation across all samples (Fig. 1a). The supervised hierarchical clustering showed that 40 miRNAs were differentially expressed when compared with control with a p-value $<3.95 \mathrm{E}-5$, and $80 \%$ were underexpressed in the OS samples. This analysis pointed out that the most underexpressed were miR-206, miR-1, miR-133a, miR-133b, all belonging to miR-206 cluster, miR-378*, and miRplus-c1049, that not being included in miRBase database was excluded from further analysis (Fig. 1b). When comparing low- and high-grade OS, unsupervised analysis identified 12 differentially expressed miRNAs, including miR-378* (p-value <0.05) (Fig. 1c).

miRNA expression levels in OS clinical specimens and cell lines. Levels of the most relevant miRNAs, miR-1, miR-133b and miR-378*, were determined by RT-PCR in a larger cohort including 56 OS (31 high- and 25 low-grade) and 13 normal control samples. Results confirmed a lower expression in OS than in controls for all selected miRNAs when osteoblasts are used as calibrator (value $=1$ ) (Fig. 2). In detail, miR-1 median values $\left(2^{-\Delta \Delta \mathrm{Ct}}\right)$ were 372.5 for OS samples and 422913 for control group $(\mathrm{p}=0.0005)$, miR-133b median values were 7.5 for OS and 2619 for control $(p=0.0005)$, miR-378* median values were 133 in OS and 1681 in control group $(\mathrm{p}<0.001)$.

In addition, within tumor population, slightly lower median values were found in high-grade as compared with low-grade OS for miR-1, miR-133b and miR-378* (Table II), and in metastatic tumors as compared to non-metastatic ones 
Table I. Clinical characteristics of patients.

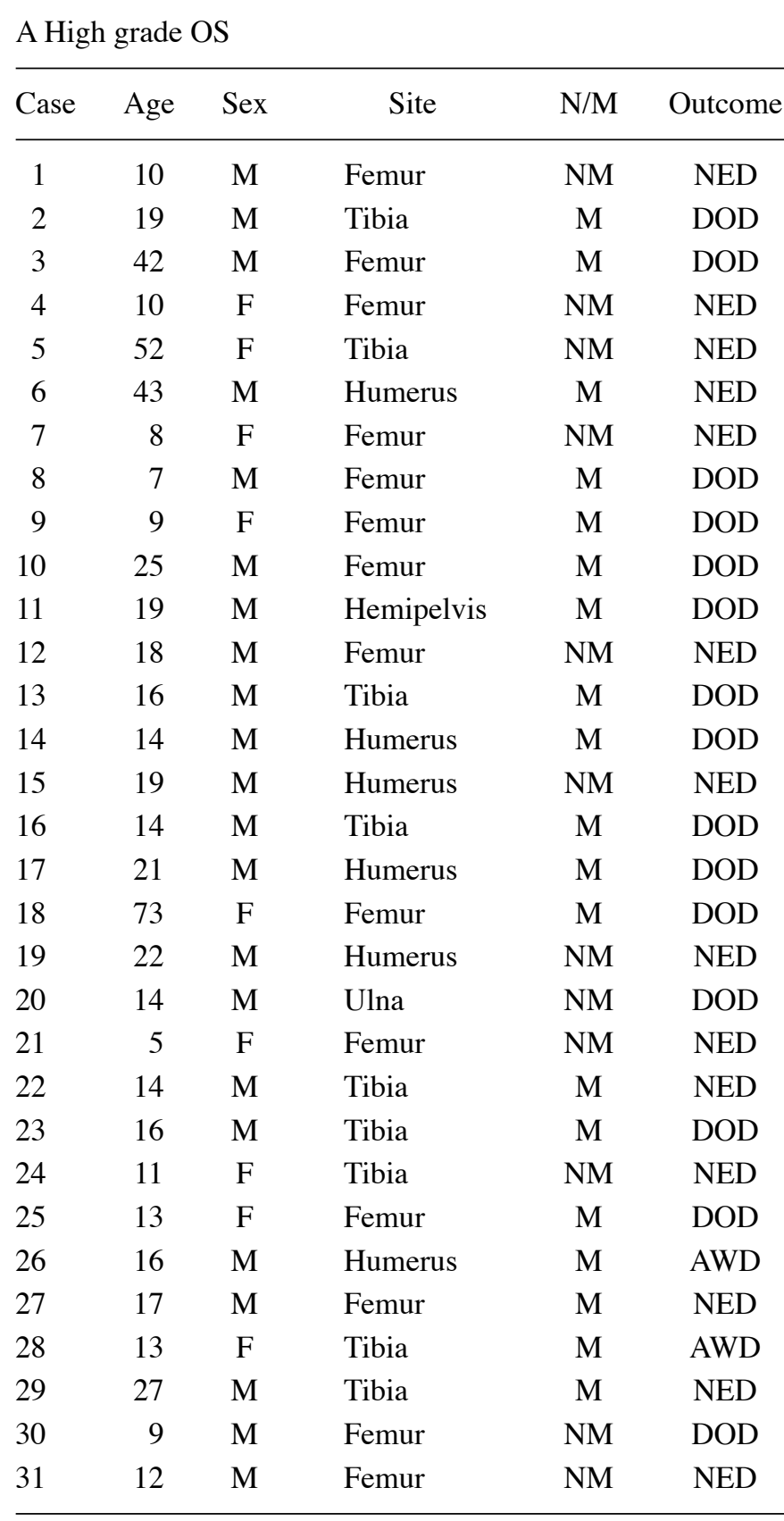

(294 vs 605 for miR-1; 5 vs 45 for miR-133b; and 103 vs 162 for miR-378*), with no statistical differences.

The expression of miR-1 and miR-133b, that resulted significantly lower in OS than in normal tissue, was investigated in OS cell lines and normal osteoblasts using MSC as calibrator. RT-PCR data showed that U2-OS, MG-63 and SAOS had, respectively, 300-, 27- and 35-fold lower miR-1 expression and 5-, 17- and 12-fold lower miR-133 expression when compared to normal osteoblasts (Table III).

Influence of miR-1 and miR133b on OS cell behavior and cell cycle distribution. To test whether increased levels of miR-1 and miR-133b might affect OS cell behavior, we introduced pre-miR miRNA precursor molecules in U2-OS cells. Transfection was validated by RT-PCR that assessed
Table I. Continued.

B Low grade OS

\begin{tabular}{ccclcc}
\hline Case & Age & Sex & \multicolumn{1}{c}{ Site } & N/M & Outcome \\
\hline 1 & 43 & M & Femur & NM & NED \\
2 & 32 & M & Femur & NM & NED \\
3 & 18 & M & Tibia & M & AWD \\
4 & 36 & F & Humerus & NM & NED \\
5 & 23 & M & Femur & NM & NED \\
6 & 26 & M & Metatarsus & NM & NED \\
7 & 53 & F & Femur & NM & NED \\
8 & 30 & M & Tibia & NM & NED \\
9 & 39 & F & Femur & NM & NED \\
10 & 23 & M & Femur & NM & NED \\
11 & 24 & F & Femur & NM & NED \\
12 & 32 & F & Humerus & NM & NED \\
13 & 21 & F & Femur & NM & NED \\
14 & 11 & F & Metatarsus & NM & NED \\
15 & 36 & F & Metacarpus & NM & NED \\
16 & 27 & F & Radio & NM & NED \\
17 & 26 & F & Femur & NM & NED \\
18 & 30 & F & Knee & NM & NED \\
19 & 43 & F & Sovracetabular & NM & NED \\
20 & 14 & F & Femur & NM & NED \\
21 & 23 & F & Humerus & NM & NED \\
22 & 22 & M & Humerus & NM & NED \\
23 & 39 & F & Femur & NM & NED \\
24 & 18 & F & Femur & NM & NED \\
25 & 13 & M & Humerus & M & AWD \\
\hline
\end{tabular}

$\mathrm{M}$, metastatic; NM, non-metastatic; NED, no evidence of disease; DOD, died of disease; AWD, alive with disease.

increased $\Delta \mathrm{Ct}$ levels in transfected cells when compared with scramble and U2-OS wt used as controls (Fig. 3a and b) and by flow cytometry.

With a transfection efficiency over $70 \%$ we found that the ectopic expression of miR-133b and miR-1 progressively decreased cell proliferation up to $72 \mathrm{~h}$ of transfection (67 and $74 \%$ of control, respectively) (Fig. 3c). Concomitantly, by Annexin V-FITC assay, induction of cell death, more evident in miR-133b transfected cells $(\mathrm{p}<0.05)$, was seen when compared to controls (Fig. 3d). The invasion analysis by Boyden chamber and migration through polycarbonate membrane showed that ectopic expression of miR-133b and miR-1 reduced cell invasiveness up to $48 \mathrm{~h}$ of 15 and 29\%, respectively $(\mathrm{p}<0.05)$ (Figs. $4 \mathrm{a}$ and $5 \mathrm{a})$ and cell migration of $22 \%(\mathrm{p}<0.05)$ and $43 \%(\mathrm{p}<0.01)$ (Figs. $4 \mathrm{~b}$ and $5 \mathrm{~b})$. However, at $72 \mathrm{~h}$ the percentage of invasive and migrating cells shifted towards control levels.

Cell cycle distribution revealed that exposure of U2-OS to miR-133b induced a transient cell accumulation in $\mathrm{S}$ and 
a
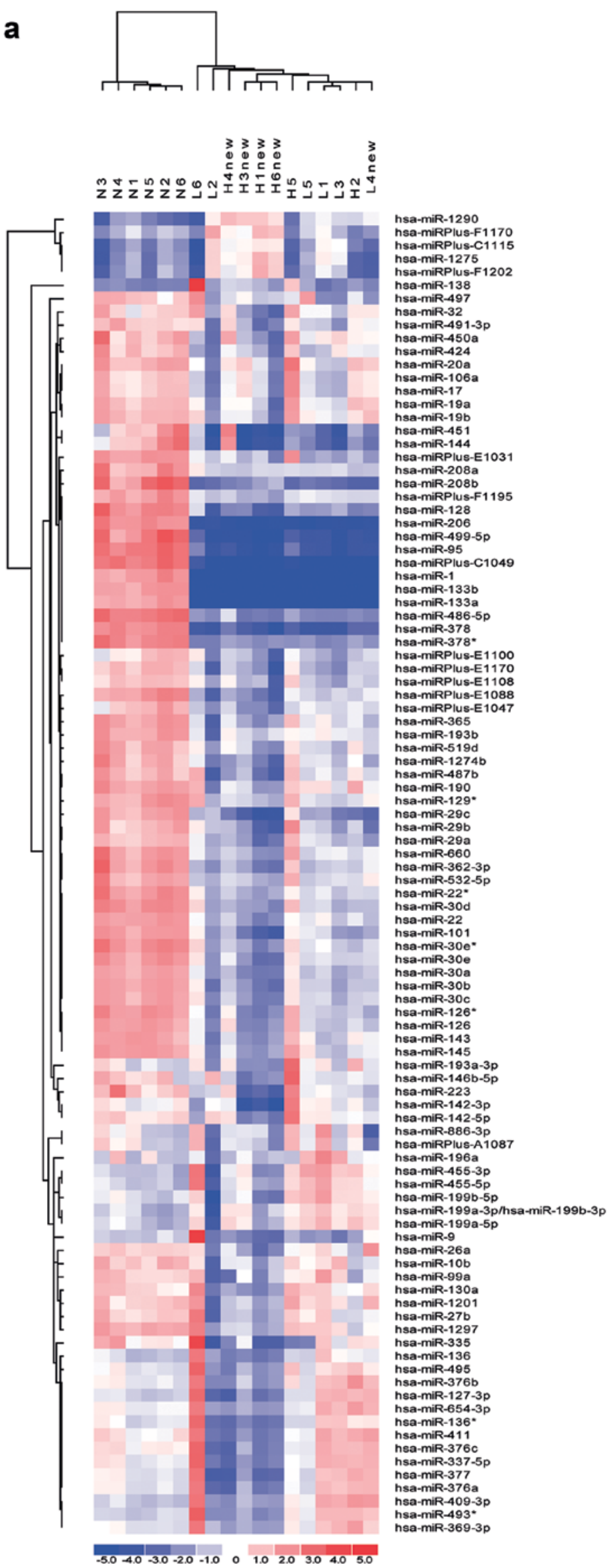

b
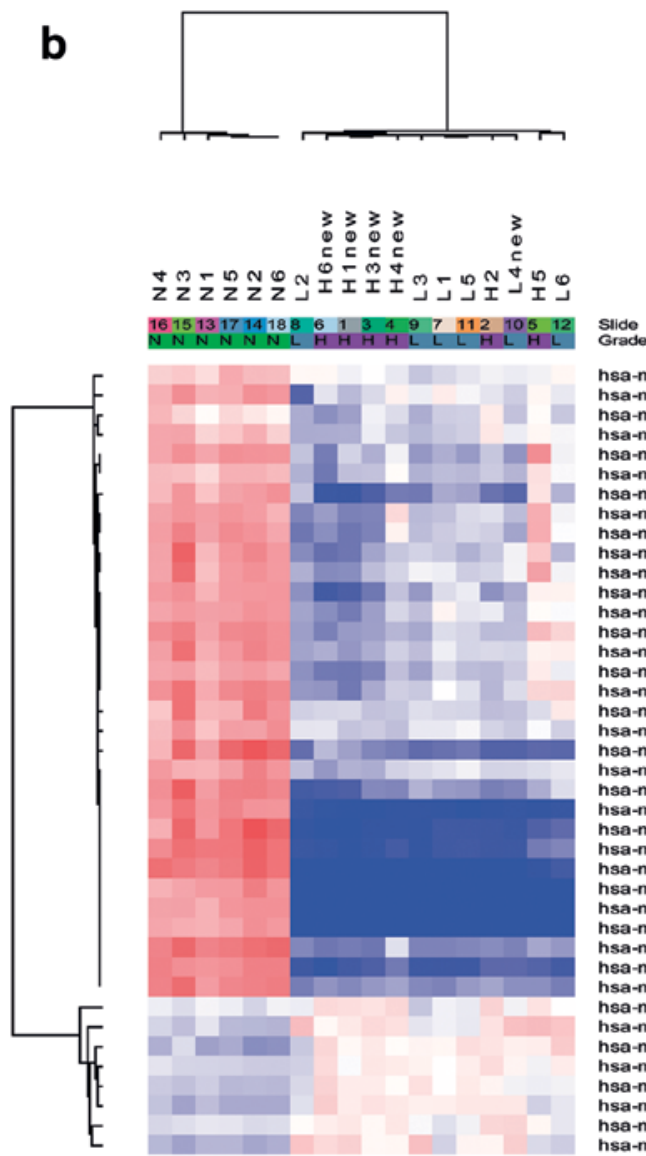

hsa-miRPlus-E1076 hsa-miR-193b hsa-miR-1280 hsa-miRPlus-E1031 hsa-miRPlus-E1172 hsa-miR-29c hsa-miR-126. hsa-miR-126 hsa-miR-362-3p hsa-miR-101 hsa-miR-22 hsa-miR-30d hsa-miR-22" hsa-miR-30a hsa-miR-2080 hsa-miR-208a
hsa-miR-29c* hsa-miR-208b hsa-miRPlus-F1195 hsa-miR-128 hsa-miR-206 hsa-miR-499-5p hsa-miRPlus-C1049 hsa-miRPlushsa-miR-1 hsa-miR-133a hsa-miR-486-5p hsa-miR-378 hsa-miR-378* hsa-miRPlus-E1029 hsa-miRPlus-E1196 hsa-miRPlus-A106 hsa-miR-342-5 hsa-miRPlus-E1112 hsa-miR-1285 hsa-miRPlus-E1026

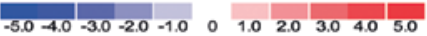

C
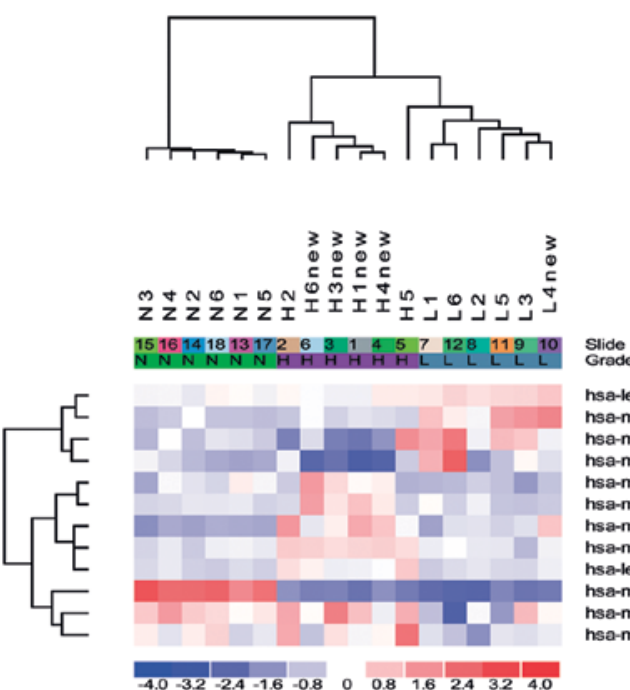

\section{(1)} hsa-miR-34c-5p hsa-miR-34a hsa-miR-136 hsa-miR-1272 hsa-miRPlus-E120s hsa-miRPlus-E102 hsa-let-7a-2*
hsa-miR-378* hsa-miR-422a

$40-32-24-16-08$ 0 $08+16243240$

Figure 1. The heat map diagram shows results of the hierarchical clustering of miRNAs and samples. The color scale shows the relative expression level of miRNA across all samples, in red expression levels above the mean, in blue expression below the mean. (a) Top 100 miRNAs with highest standard deviations across all samples by unsupervised analysis. (b) 40 miRNAs differentially expressed between tumor and control by supervised analysis (p<3.95E-5). (c) 12 miRNAs differentially expressed between high $(\mathrm{H})$, low $(\mathrm{L})$ grade $\mathrm{OS}$ and normal $(\mathrm{N})$ tissue by unsupervised analysis $(\mathrm{p}<0.05)$. 

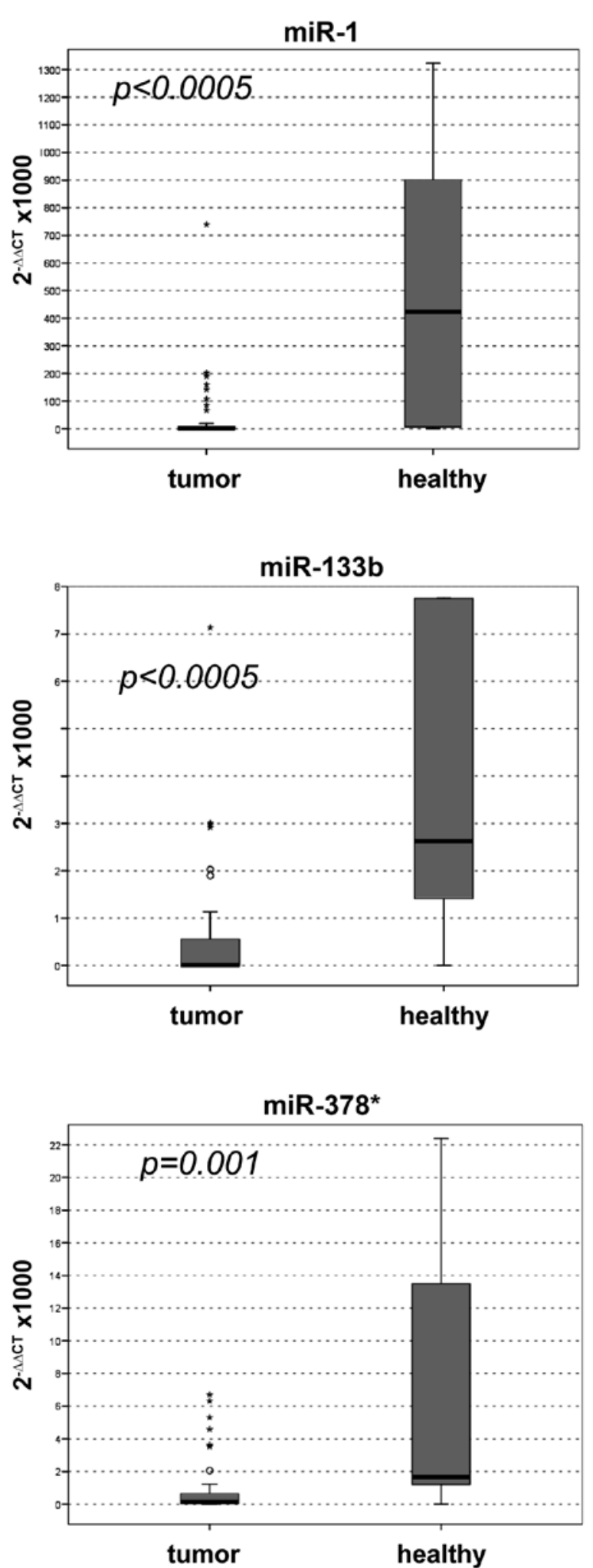

Figure 2. Non-Gaussian distribution showed that miR-1, miR-133b and miR-378* mRNA values were lower in primary OS than in healthy tissue. Mann-Whitney U test revealed statistically significant differences between the two groups for all miRNAs studied.

$\mathrm{G}_{2}$ phase up to $48 \mathrm{~h}$ (Fig. $4 \mathrm{c}$ ), while miR-1 ectopic expression resulted in accumulation of cells in $\mathrm{G}_{1}$ phase coincident with a cell decrease in $\mathrm{S}$ phase up to $48 \mathrm{~h}$ of transfection (Fig. $5 \mathrm{c}$ ), suggesting a block at $\mathrm{G}_{1} / \mathrm{S}$ check point.
Table II. miRNA expression in high and low grade OS.

\begin{tabular}{|c|c|c|c|c|}
\hline & Median $2^{-\triangle \Delta C T}$ & $25 \mathrm{P}$ & $75 \mathrm{P}$ & $\mathrm{p}$-value \\
\hline \multicolumn{5}{|l|}{ miR-1 } \\
\hline High & 346 & 45.50 & $6,835.50$ & NS \\
\hline Low & 400 & 27 & $18,951.00$ & \\
\hline \multicolumn{5}{|c|}{ miR-133b } \\
\hline High & 7 & 1.00 & 598.50 & NS \\
\hline Low & 8 & 2.00 & 662.00 & \\
\hline \multicolumn{5}{|c|}{ miR-378* } \\
\hline High & 127 & 26.50 & $1,112.50$ & NS \\
\hline Low & 139 & 16.00 & 600.00 & \\
\hline
\end{tabular}

Table III. miR-1 and miR-133b expression in OS cell lines and normal osteoblasts.

\begin{tabular}{lccccc}
\hline & \multicolumn{2}{c}{$\mathrm{miR}-1$} & & \multicolumn{2}{c}{$\mathrm{miR}-133 \mathrm{~b}$} \\
\cline { 2 - 3 } \cline { 6 - 6 } & $\left(2^{-\Delta \Delta \mathrm{CT}}\right)$ & $\mathrm{n}$-fold & & $\left(2^{-\Delta \Delta C \mathrm{C}}\right)$ & $\mathrm{n}$-fold \\
\hline Osteoblasts & $2.40 \mathrm{E}-01$ & & & 11.63 & \\
U2-OS & $8.00 \mathrm{E}-04$ & 300 & & 2.31 & 5 \\
MG-63 & $9.00 \mathrm{E}-03$ & 27 & & $6.60 \mathrm{E}-01$ & 17 \\
SAOS & $7.00 \mathrm{E}-03$ & 35 & & $9.30 \mathrm{E}-01$ & 12 \\
\hline
\end{tabular}

miR-1 and miR133b negatively regulate MET protein expression. To investigate the molecular mechanism of the anti-proliferative propriety of miR-1 and miR-133b, we examined the expression of oncogene Met in U2-OS transfected cells that previous data indicated as a candidate target of both miRNAs $(15,16)$. Relative to non-transfected cells and scramble, ectopic expression of both miR-1 and miR-133b led to a reduced expression of MET protein level (Fig. 6). Adjusted volume of the electrophoretic bands quantified by densitometric analysis was 21.83 and 17.52, respectively, for controls, 5.04 for miR-1 and 8.43 for miR-133b transfected cells. However, Met gene mRNA levels were not different in miRNA-1 and miR-133b transfected U2-OS as compared with negative control $(\Delta \mathrm{Ct}=6.40$ vs 6.30 and 5.20 vs 4.73 , respectively), suggesting an incomplete pairing between miRNA and mRNA target.

\section{Discussion}

The molecular characterization of OS shows a genetic complexity that has a limited value in diagnosis and prognosis. In addition, the data emerging from clinical studies show that $35-45 \%$ of OS patients have a natural or acquired drug resistance (17). It is, thus, clear that the importance of identification of specific predictive and prognostic biomarkers allow a better stratification of patients and a more precise evaluation of risk. Recent studies emphasized the 

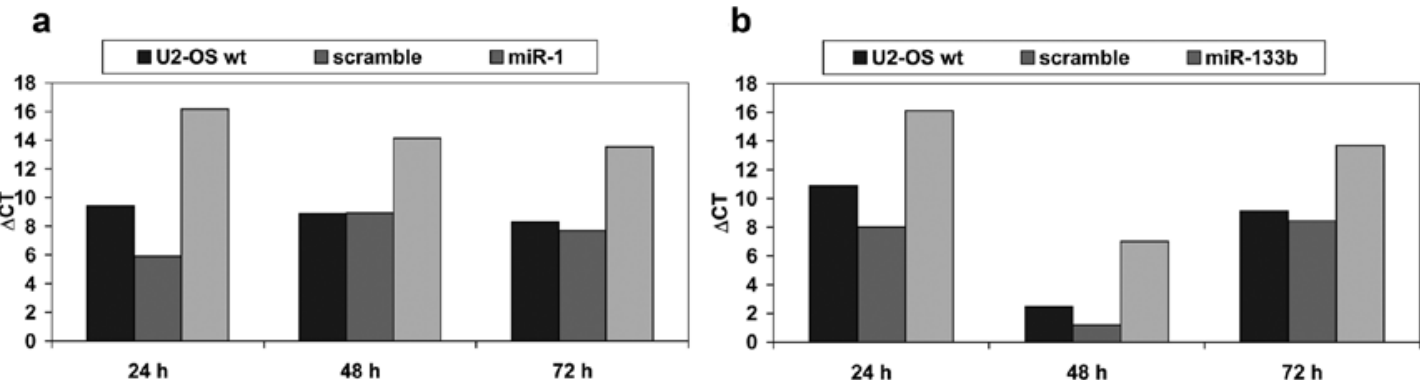

c

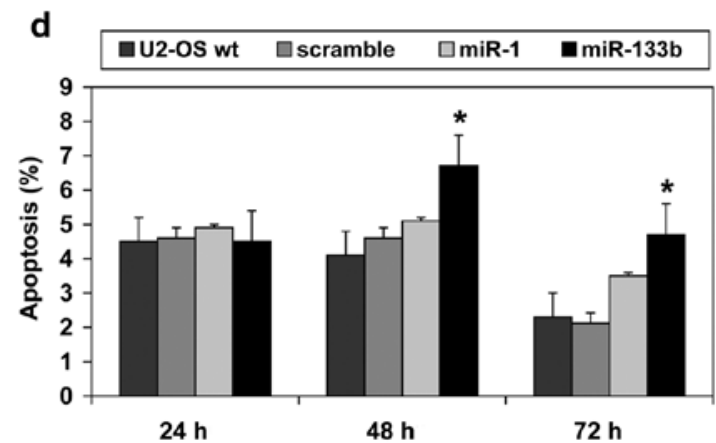

Figure 3. Efficiency of transfection with (a) pre-miR miR-1 precursor and (b) pre-miR miR-133b precursor in U2-OS cells by using real-time PCR ( $\Delta \mathrm{Ct}$-values). Ectopic miR-1 and miR-133b causes (c) decrease in U2-OS cell proliferation, and (d) increase in apoptotic fraction after 48-72 $\mathrm{h}$ of transfection when compared to controls (U2-OS wt and scramble). ${ }^{\mathrm{p}}<0.05$.
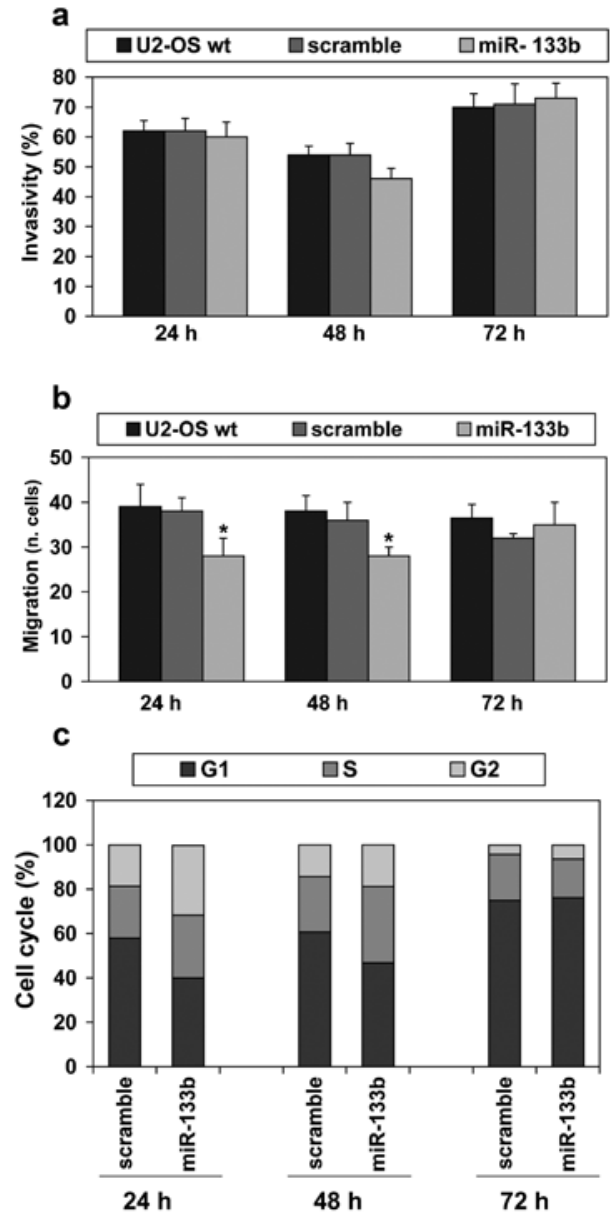

Figure 4. Effect of ectopic miR-133b on U2-OS cells. (a) Cell invasion and (b) migration assays show reduction in invasive ability and motility up to $48 \mathrm{~h}$ as compared to U2-OS wt and scramble. (c) Cell cycle distribution reveals transient cell accumulation in S/G2 phase up to $48 \mathrm{~h}$ of transfection. " $\mathrm{p}<0.05$.
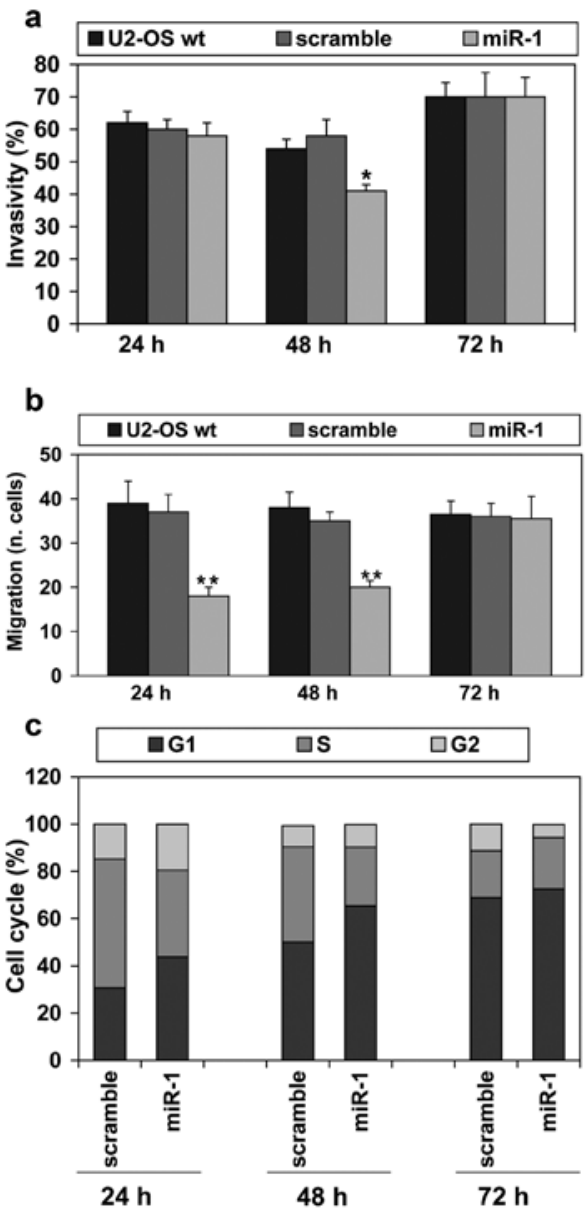

Figure 5. Effect of ectopic miR-1 on OS cells. (a) Invasion and (b) migration assays show decrease of invasiveness and motility up to $48 \mathrm{~h}$ as compared to controls. (c) Cell cycle distribution reveals cell accumulation in $\mathrm{G}_{1}$ phase, with decrease in $\mathrm{S}$ phase up to $48 \mathrm{~h}$ of transfection. ${ }^{*} \mathrm{p}<0.05,{ }^{* *} \mathrm{p}<0.01$. 


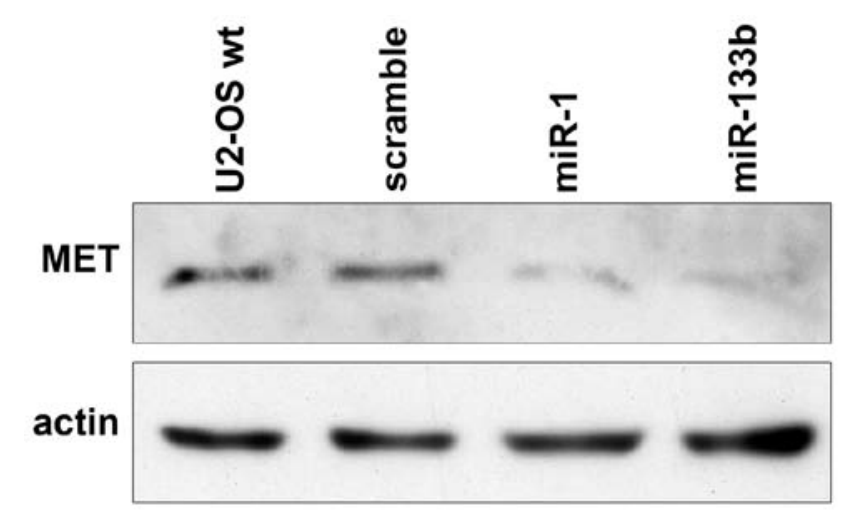

Figure 6. MET protein expression in U2-OS cells by western blot analysis. Cells transfected with miR-1 and miR-133b show less intense migrating band when compared to U2-OS wt and scramble. Control loading is shown by actin.

role of deregulated miRNAs in increasing viability, invasion and proliferation of OS cells, investigating their activity in affecting chemoresistance (18-20).

In our OS samples miRNA expression profiling showed 40 miRNAs deregulated with respect to normal samples and 12 differentially expressed across OS groups. Some miRNAs identified appear related to cancer development. miR-22 is involved on PTEN/AKT pathway (21), miR-95 promotes cell proliferation in colorectal carcinoma (22), miR-30a is involved in cell invasion mechanism (23) and miR-378 is a negative regulator of $\mathrm{SuFu}$, an oncosuppressor gene essential for cell survival (24). The following validation of the most significant miRNAs in a larger series of human OS samples and cells lines confirmed a significant lower expression of miR-1 and miR-133b when compared with normal tissue, although their deregulation does not seem to be involved in progression towards high histological grade.

Chen et al (25) characterized cardiac-specific and skeletal muscle-specific miR-1 and miR-133b and showed their essential function in controlling skeletal muscle proliferation and differentiation. The impact of ectopic miR-1 and miR-133 expression on OS cells behavior was analyzed in U2-OS cell lines, showing reduced proliferative capacity when compared with controls and transient perturbations in cell cycle in agreement with the idea that some microRNAs function within pathways that control cell cycle checkpoints (26).

Cells transfected with the precursor of miR-1 showed a transient block of cell cycle in $\mathrm{G}_{1}$ phase, concordant with cell decrease in $\mathrm{S}$ phase up to $48 \mathrm{~h}$ of transfection and without significant induction of apoptosis. In contrast, cell lines treated with the precursor of miR-133b showed accumulation of cells at $S / G_{2}$ phase associated with decrease in $G_{1}$ phase up to $48 \mathrm{~h}$ of exposure. This prolongation of $\mathrm{S} / \mathrm{G}_{2}$ phase without cell cycle arrest is accompanied by significant induction of apoptosis supporting previous observations that identified anti-apoptotic proteins as predicted targets for miR-133b (27).

Interestingly, in our study OS cells responded to ectopic expression of both miR-1 and miR-133b significantly decreasing cell invasion and motility in short-term assays. These data agree with recent studies in rhabdomyosarcoma where transfection of miRNAs caused block of the cell cycle progression in $G_{1}$ and decrease in cell proliferation and
Matrigel invasiveness through modulation of global gene expression profile (28). In addition, miR-1 was shown to have activity on mRNA expression by targeting Met 3' untranslated region, thus proposing $M e t$ as target gene (15).

Concomitantly, ectopic expression miR-133b potently affected colorectal cancer cell proliferation and apoptosis in vitro and in vivo by direct targeting of the receptor tyrosine kinase MET (16). To explore if miR-1 and miR-133b regulate OS tumor cell proliferation through modulation of the $\mathrm{Met}$ signaling pathway, we analyzed MET protein by western blot analysis, and confirmed that the forced expression of both led to a significant decrease of its expression levels. Conventional therapy for OS has reached a plateau of survival in the past 20 years, highlighting the need for new therapeutic approaches. Thus, the identification of a list of candidate miRNAs controlling signaling pathways might overcome the genomic complexity of this tumor improving prognosis and drug-responsiveness. In particular, our data suggest a potential anti-proliferative and anti-invasive function of miR-1 and miR-133b in OS cells through modulation of the MET protein expression.

\section{Acknowledgements}

The authors wish to thank Dr Alba Balladelli for editing the manuscript, Ms. Cristina Ghinelli for her graphic work, Dr Roberto Mirabella and Dr Paola Poggi for statistical support. This study was supported by Istituto Superiore della Sanita (ITALIA-USA project) and 5\%o donation (Italy).

\section{References}

1. Gorlick R and Khanna C: Osteosarcoma. J Bone Miner Res 25: 683-691, 2010.

2. Gougelet A, Pissaloux D, Besse A, et al: Micro-RNA profiles in osteosarcoma as predictive tool for ifosfamide response. Int J Cancer 129: 680-690, 2011.

3. John B, Enright AJ, Aravin A, et al: Human MicroRNA targets. PloS Biol 2: e363, 2004.

4. Lagos-Quintana M, Rauhut R, Yalcin A, et al: Identification of tissue-specific microRNAs from mouse. Curr Biol 12: 735-39, 2002.

5. Lee RC and Ambros V: An extensive class of small RNAs in Caenorhabditis elegans. Science 294: 862-64, 2001.

6. Sempere LF, Freemantle S, Pitha-Rowe I, et al: Expression profiling of mammalian microRNAs uncovers a subset of brain-expressed microRNAs with possible roles in murine and human neuronal differentiation. Genome Biol 5: R13, 2004.

7. Lim LP, Lau NC, Weinstein EG, et al: The microRNAs of Caenorhabditis elegans. Genes Dev 17: 991-1008, 2003.

8. Esquela-Kerscher A and Slack FJ: Oncomirs - microRNAs with a role in cancer. Nat Rev Cancer 6: 259-269, 2006.

9. Calin GA, Sevignani C, Dumitru CD, et al: Human microRNA genes are frequently located at fragile sites and genomic regions involved in cancers. Proc Natl Acad Sci USA 101: 2999-3004, 2004.

10. Wu W, Sun M, Zou GM and Chen J: MicroRNA and cancer: current status and prospective. Int J Cancer 120: 953-960, 2007.

11. Montanini L, Lasagna L, Barili V, et al: MicroRNA cloning and sequencing in osteosarcoma cell lines: differential role of miR-93. Cell Oncol (Dordr) 35: 29-41, 2012.

12. He C, Xiong J, Hu X, et al: Functional elucidation of MiR-34 in osteosarcoma cells and primary tumor samples. Biochem Biophys Res Commun 388: 35-40, 2009.

13. Kauppinen S, Vester B and Wengel J: Locked nucleic acid: high-affinity targeting of camplementary RNA for RNomics. Handb Exp Pharmacol 173: 405-422, 2006.

14. Yang YH, Dudoit S, Luu P, et al: Normalization for cDNA microarray data :a robust composite method addressing single and multiple slide systematic variation. Nucleic Acids Res 30: $\mathrm{e} 15,2002$. 
15. Yan D, Dong Xda E, Chen X, et al: MicroRNA-1/206 targets c-Met and inhibits rhabdomyosarcoma development. J Biol Chem 284: 29596-29604, 2009.

16. $\mathrm{Hu} \mathrm{G}, \mathrm{Chen} \mathrm{D}, \mathrm{Li}$ X, et al: miR-133b regulates the MET protooncogene and inhibits the growth of colorectal cancer cells in vitro and in vivo. Cancer Biol Ther 10: 190-197, 2010.

17. Hattinger CM, Pasello M, Ferrari S, et al: Emergine drugs for high-grade osteosarcoma. Export Opin Emerg Drugs 15 615-634, 2010

18. Zhang H, Cai X, Wang Y, et al: microRNA-143, down-regulated in osteosarcoma, promotes apoptosis and suppresses tumorigenicity by targeting Bcl-2. Oncol Rep 24: 1363-1369, 2010.

19. Ziyan W, Shuhua Y, Xiufang W and Xiaoyun L: MicroRNA-21 is involved in osteosarcoma cell invasion and migration. Med Oncol 28: 1469-1474, 2011

20. Song B, Wang Y, Titmus MA, et al: Molecular mechanism of chemoresistance by miR-215 in osteosarcoma and colon cancer cells. Mol Cancer 9: 96, 2010.

21. Bar N and Dikstein R: MiR-22 forms a regulatory loop in PTEN/AKT pathway and modulates signaling kinetics. PloS One 27: e10859, 2010.

22. Huang Z, Huang S, Wang Q, et al: MicroRNA-95 promotes cell proliferation and targets sorting Nexin 1 in human colorectal carcinoma. Cancer Res 71: 2582-589, 2011.
23. Kumarswamy R, Mudduluru G, Ceppi P, et al: MicroRNA-30a inhibits epithelial-to-mesenchymal transition by targeting Snail and is downregulated in non small cell lung cancer. Int J Cancer 130: 2044-2053, 2012.

24. Lee DY, Deng Z, Wang CH and Yang BB: MicroRNA-378 promotes cell survival, tumor growth, and angiogenesis by targeting SuFu and Fus-1 expression. Proc Natl Acad Sci USA 104: 20350-20355, 2007.

25. Chen JF, Mandel EM, Thomson JM, et al: The role of microRNA-1 and microRNA-133 in skeletal muscle proliferation and differentiation. Nat Genet 38: 228-233, 2006.

26. Carleton M, Cleary MA and Linsley PS: MicroRNAs and cell cycle regulation. Cell Cycle 6: 2127-2132, 2007.

27. Crawford M, Batte K, Yu L, et al: MicroRNA 133B targets pro-survival molecules MCL-1 and BCL2L2 in lung cancer. Biochem Biophys Res Commun 388: 483-489, 2009.

28. Taulli R, Bersani F, Foglizzo V, et al: The muscle-specific microRNA miR-206 blocks human rhabdomyosarcoma growth in xenotransplanted mice by promoting myogenic differentiation. J Clin Invest 119: 2366-2378, 2009. 